\title{
Research on Target Area Mechatronics System for ICF Laser Driver Wang Liquan ${ }^{1}$, Jing Yukun ${ }^{1}$, Xiang Yong ${ }^{1, C}$, Feng Bing ${ }^{1}$, Han Wei ${ }^{1}$, Li Fuquan ${ }^{1}$, Wang Fang ${ }^{1}$, Li Keyu ${ }^{1}$ \\ ${ }^{1}$ Research Center of Laser Fusion CAEP, Mianyang, China \\ c email: wanglq1013@caep.cn , Correspondence: Tel: +86-18081247627
}

Keywords: Mechatronics System; Target Area; ICF; Laser Driver; beams precisely leading; target precisely fixed position; shooting target practice accuracy

\begin{abstract}
This paper will introduce in detail how to adopt the Target Area Mechatronics System to realize the full closed loop control of light beam leading and target precisely fixed position, and Target Chamber Vacuum Control System and Ambi-room Sweeping Control System, auto program flow of shooting target practice process by Target Area Integrated Control System, make the system shooting practice preparative time less than 30 minutes and shooting target practice accuracy better than $30 \mu \mathrm{m}$ (RMS). Its performance superiority will decide operation efficiency and success or failure of physics experiment on the whole ICF laser driver facility.
\end{abstract}

\section{Background}

Using deuterium and tritium of hydrogen's isotope for the hot nuclear fusion is always thought the most valid method that can solve energy problem facing mankind for a long time. But the high power pulse laser (quantity class about the $1014 \mathrm{~W}$ ) from the short-wave length, namosecond laser driver is currently accepted as the most hopeful technique to realize deuterium and tritium fusion combustion (ICF means inertial confinement fusion). Therefore, the United States, Russia, Japan, France, Germany, English etc. are aggressively busy in carrying out research on this scientific field one after the other. Energy Department of the United States has planned to realize the demonstration experiment of the fusion ignition by using this technique (as Fig. 1 shows).
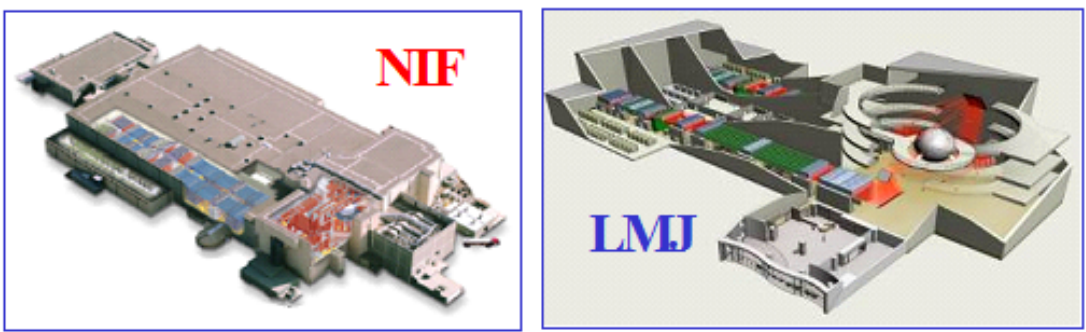

Fig. 1 American NF (Nation Ignition Facility) and France LMJ(Laser Megaj oule Facility)

\section{Introduction}

As one great high power solid laser facility applied on ICF research field in China, ShenGuangIII prototype facility can produce eight triple-frequency laser beams up to ten thousand joule. And each light beam's caliber is $290 \mathrm{~mm} \times 290 \mathrm{~mm}$. Fig, 2 shows constitute principle sketch map of ShenGuang- III prototype facility. The laser facility includes front-end, pre-amplifier, disk-amplifier, main-amplifier, Diagnostic unit and Target area etc. system. Among them, as an important direct interface with physics experiment, target area locates in the end of the whole facility. From the main amplifier class output port to the target point, it has more than 30 miles long, and includes 5 pieces or 7 pieces transfers mirrors, a Final Optical Module FOM to realize a series function including transfers of the main lasers, triple-frequency wave conversion and the accurate target position etc. (as Fig. 2 shows). 


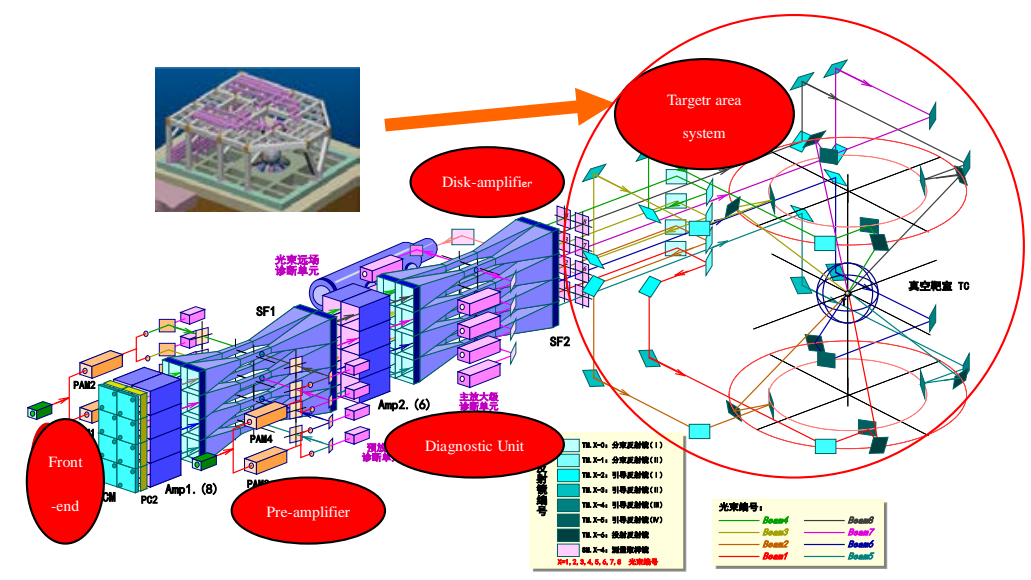

Fig.2 constitute principle sketch map of ShenGuang-III prototype facility

\section{Basic function and constitutes of TAS}

At first, it is the main mission of TAS (Target Area System) to provide the experiment terrace for validating lasers synchronous output and precision shooting practice function of ShenGuang-III prototype facility. And it can complete these experiment research of many-light beams precisely leading, target precisely fixed position technique, focus special distribution and focus shape modification etc. Moreover, it can make certain whether laser beams shooting target practice could satisfy physics experiment' request. And it could provide enough important design data and basis for the concept design of ShenGuang-III host facility. Doubtless, as the most important experiment
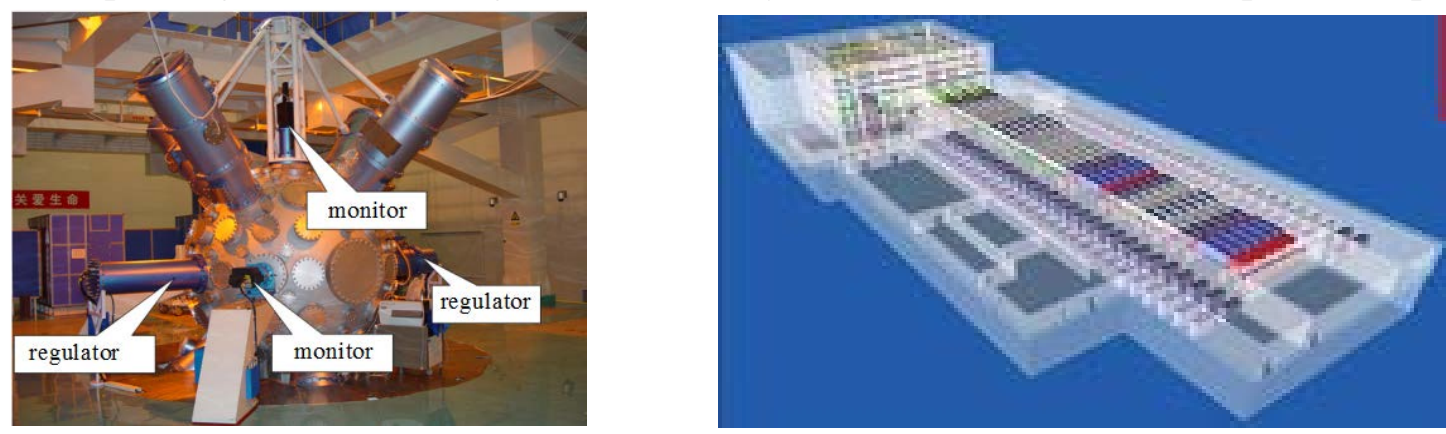

Fig.3 Real object photograph of TAS on prototype facility and concept design of host facility terrace and field for physics experiment on prototype facility, TAS must surely satisfy each changefully physics experiment request. TAS has become one core constitute part of the ICF laser driver facility (as Fig, 3 shows).

\begin{tabular}{|l|l|l|l|l|l|l|l|}
\hline \multicolumn{7}{|c|}{ TAS } \\
\hline BTS & \multicolumn{1}{|l|}{} & & \\
\hline FOM & TC & ASC & TPS \\
\hline
\end{tabular}

Fig. 4 constitution frame diagram of TAS

TAS mainly includes Beam Transmit System BTS、Final Optical Module FOM、Target Chamber TC、Ambi-room Sweeping Control System ASCS、Target Position System TPS and Target Area Integrated Control System TAICS(as Fig,4 shows), among which TPS and TAICS will be introduced respectively underneath in detail .

Target Position System TPS. For ShenGuang-III prototype facility, it adopts three high accuracy monitors and a many-light beams target surface sensor and a set of high accuracy six-dimension target regulator and a set of high accuracy six-dimension sensor regulator, as well as corresponding optoelectronics and control system including of soft and hard hardware, to carry out many-light beams precisely leading and target precisely fixed position. Concrete components and layout as figure 5 shows. The many-light beams target surface sensor is actually a monitor part to 
match between light beams and the target, which is designed by the principle of optics conjugate for detecting the gesture of physics experiment targets and the position of shooting points of many light beams. Many-light beams target surface sensor, which is core part of the many-light beams precisely leading and target precisely fixed position system, as figure 6 shows.
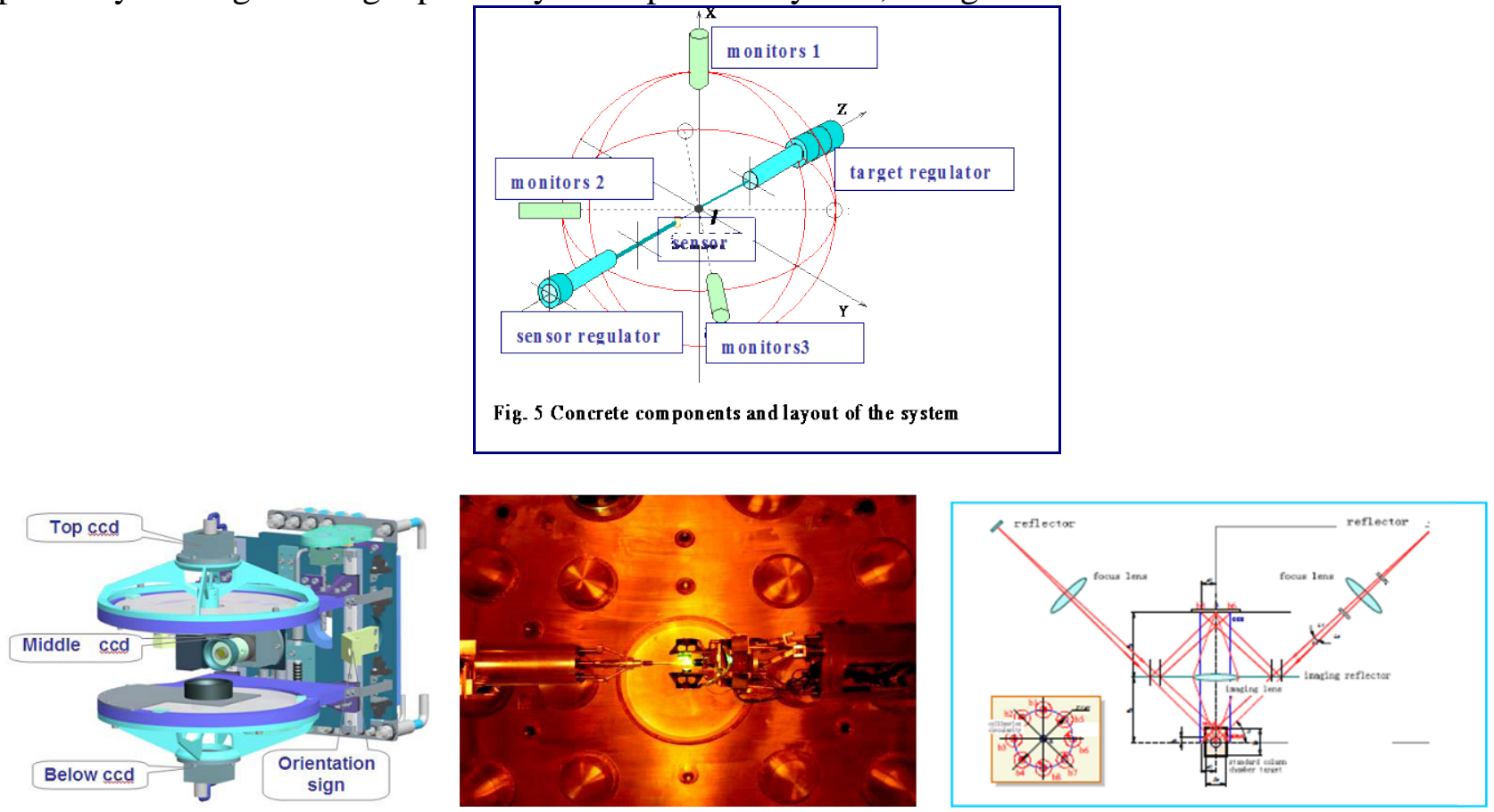

Fig. 6 Original design of the sensor, coupling beams and the target, Basic principle of beams guiding

Target Area Integrated Control System TAICS. As an importance constitute part, TAICS could carry on auto-control of the whole TA system according to work relation of different control units. TAICS has become the foundation to decide physics experiments successful or false. The technique route of TAICS is adopting three layers structure design, including remote control layer and the local control layer, the executing equipments layer. Remote control layer is made up of software and hardware environment used for integrated and remote control in host center control room. The local control layer is made up of many variety computers and control software, which is distributed on the spot used for realizing function control. The executing equipments layer is made up of motors and CCD and sensors and other electrical equipments, and so on in local machines.

\section{Workflow}

First, it must ascertain target chamber benchmark by two flanges on the plate of the target chamber equator. Moreover, the vacuum of the target chamber must raise up to $10^{-4} \mathrm{~Pa}$ by many sets of vacuum pumps and relative vacuum control system of Target Chamber. Then, using three high accuracy perpendicular monitors to accurately fix position the many-light beams target surface sensor. Then, target surface sensor positions the physical target and each laser shooting point, at last it meets the precision requirement of physical experiment through adjusting reflector and shooting practice lens. Among them, a set of high accuracy six-dimension regulator takes charge of physical experiment target's position. Main laser beams to be guided are replaced by accurate and coaxial triple frequency simulation lights, which have the same direction and shooting points on a target fixed position at the vacuum target chamber center district. It realizes full closed loop control of light beams leading and target fixed position by photoelectric and automatic control technique based on machine sense of vision. At last, Target Area Integrated Control System TAICS will start sweeping the Final Optical Module FOM and post-manage for shooting practice will begin at the same time as soon as the formal physical experiment is over. Figure7 shows primary workflow. 


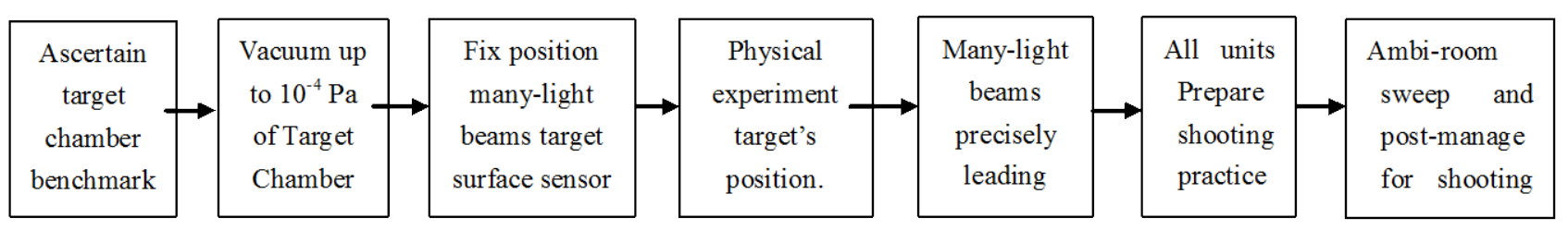

Fig. 7, Primary workflow of the system

\section{Result of the experiments}

Target Area Mechatronics System are successfully applied on ShenGuang-III prototype facility for many years. Until now, the system is stable and reliable very much, make the system shooting practice prepare time less than 30 minutes and shooting target practice accuracy better than $30 \mu \mathrm{m}$ (RMS). In order to verify shooting practice accuracy, it carry out the physical experiment of shooting thin wall chamber target and tracking record distributing situation of eight beams laser shooting points in the chamber on the prototype facility. As figure 8 shows, the left part of each photograph shows preestablishing laser shooting points, accordingly the right part shows real shooting points istributing situation obtained from by $\mathrm{X}$ light pinhole camera. It can infer that pre-establishing shooting points and real shooting points match much consistently, and the system has completely satisfied the requirement of physical experiment.
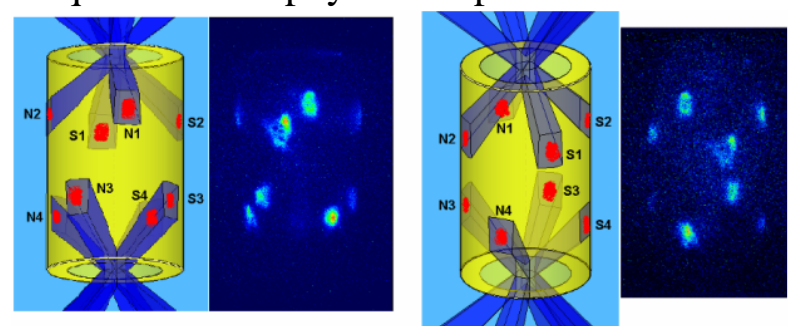

Fig. 8, Comparison pre-establishing laser shooting points with real shooting points

\section{References}

[1] LL E. The OMEGA target positioning system[R]. Rochester, 1997,145-159.

[2] Boege S J. NIF pointing and centering systems and target alignment using 351nm laser source[J]. SPIE, 1997, 3047:248-258.

[3] D G Lowe. Distinctive image features from scale invariant key points[J]. International Computer Vision, 2004, 60(2):91-110.

[4] Sun Lining, Liu Yanwu, et al. ICF target positioning robot system. High Power Laser and Particle Beams, 2007, 19(8):1303-1307.

[5] Wu Huilan, Liu Guodong, Liu Bingguo, et al. Study on location technique for ICF experiment targets. Semiconductor Optoelectronics, 2008, 29(5):774-777.

[6] Dai Yaping, Huang Guanlong, Li Xuechun. Precision target positioning by digital speckle correlation measurement. Chinese J. Lasers, 2000, A27(2):135-139. 